\title{
Zeit für Lebenslanges Lernen. Neue Ansätze der betrieblichen Arbeitszeit- und Qualifizierungspolitik
}

\author{
Philip Wotschack/Franziska Scheier \\ Philipp Schulte-Braucks/Heike Solga
}

Viele Betriebe bieten ihren Beschäftigten nicht die Möglichkeiten, sich im Laufe ihres Lebens ausreichend weiterzubilden. Zu den Ursachen liegt eine umfassende Forschung vor. Als ein wesentliches Problem erweist sich das Fehlen zeitlicher Ressourcen. Ein aktuelles Forschungsprojekt ${ }^{1}$ am Wissenschaftszentrum Berlin für Sozialforschung (WZB) untersucht, unter welchen Bedingungen Betriebe sich anders verhalten und im Rahmen der Arbeitszeitgestaltung Zeiträume für Weiterbildung bereitstellen. Das Beispiel dieser Betriebe zeugt davon, dass Alternativen zur vorherrschenden betriebswirtschaftlichen Praxis möglich sind. Es wirft die Frage nach der Art dieser Lösungen auf sowie den betrieblichen und überbetrieblichen Voraussetzungen ihres Erfolgs. ${ }^{2}$

\section{Einleitung}

Die Wichtigkeit betrieblicher Weiterbildung ist unumstritten. Für Beschäftigte bildet sie eine entscheidende Grundlage, um mit den sich schnell wandelnden technologischen, inhaltlichen und organisatorischen Anforderungen der Arbeitswelt Schritt zu halten oder sich beruflich weiterzuentwickeln. Sie erhöht die Chancen für eine kontinuierliche und langfristige Beschäftigung sowie beruflichen Aufstieg. Für Betriebe verbessert Weiterbildung die Produktivität und Qualität der Arbeitsabläufe und schützt zugleich vor der Gefahr eines langfristigen Fachkräftemangels. Letzterer wird vor dem Hintergrund der demografischen Entwicklung als ein wachsendes Risiko wahrgenommen (vgl. Morschhäuser et al. 2008).

Das Gelingen von Weiterbildung ist mit einer Reihe von Ressourcen und Rahmenbedingungen - auf betrieblicher wie individueller Seite - verbunden. Dazu zählen finanzielle Mittel, Zeit, die Motivation der Teilnehmenden, die Organisation und Qualität der Maßnahmen sowie die Anerkennung der Bildungsergebnisse (Heidemann 2010). Die Bereitstellung ausreichender zeitlicher Spielräume erweist sich dabei als eine wichtige Voraussetzung, die in der betrieblichen Praxis oft nicht gegeben ist (Dobischat/Seifert 2007; Expertenkommission 2004, S. 34; Lenske/ Werner 2009). Auf Seiten der Betriebe wie der Beschäftigten stellen fehlende zeitliche Spielräume häufig eine zentrale Ursache für fehlende Weiterbildungsaktivität dar.

$\mathrm{Zu}$ den Ursachen fehlender zeitlicher Ressourcen für betriebliche Weiterbildung existiert bereits eine umfangreiche Forschung. Kaum untersucht sind hingegen Strategien und Voraussetzungen einer Überwindung dieser „Zeit-Barrieren“. Zu selten wird der Blick auf Betriebe gerichtet, die sich anders verhalten und selbst in Phasen großen ökonomischen Drucks nachhaltige Konzepte der Arbeitszeit- und Qualifizierungspolitik verfolgen. Wie lösen sie das „Zeitressourcen-Problem“? Das heißt, wie und unter welchen Bedingungen lässt sich im Rahmen der betrieblichen Arbeitszeitgestaltung ausreichend Zeit für Weiterbildung sicherstellen? Diese Frage ist leitend für das Projekt „Betriebliche Arbeitszeit- und Qualifizierungspolitik im Lebensverlauf" am Wissenschaftszentrum Berlin für Sozialforschung (WZB) (vgl. Fußnote 1). In seinem Zentrum steht weniger das bekannte Problem fehlender zeitlicher Ressourcen für Weiterbildung, als vielmehr die Suche nach möglichen Wegen und Voraussetzungen, um diese Barriere für Weiterbildungsaktivitäten zu überwinden.

Das Projekt geht in drei analytischen Schritten vor. In einem ersten Schritt wur-

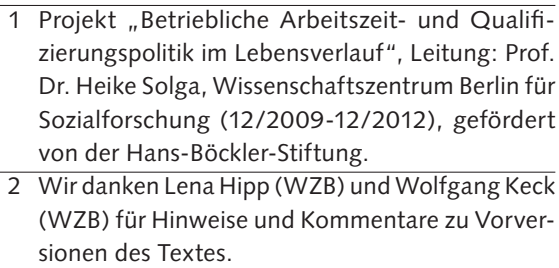

1 Projekt „Betriebliche Arbeitszeit- und Qualifizierungspolitik im Lebensverlauf“", Leitung: Prof. Dr. Heike Solga, Wissenschaftszentrum Berlin für Sozialforschung (12/2009-12/2012), gefördert von der Hans-Böckler-Stiftung.

2 Wir danken Lena Hipp (WZB) und Wolfgang Keck (WZB) für Hinweise und Kommentare zu Vorversionen des Textes.

Philip Wotschack, Dr., Soziologe,

Wissenschaftlicher Mitarbeiter der Abteilung Ausbildung und Arbeitsmarkt am Wissenschaftszentrum Berlin für Sozialforschung (WZB). Arbeitsschwerpunkte: Arbeitszeit und Arbeitsorganisation, Betriebliche Weiterbildung, Vereinbarkeit von Beruf und Familie. e-mail:wotschack@wzb.eu Franziska Scheier, Dipl.-Sozialwissenschaftlerin, Stipendiatin der Hans-Böckler-Stiftung und Gastwissenschaftlerin der Abteilung Ausbildung und Arbeitsmarkt am WZB. Arbeitsschwerpunkte: Betriebliche Arbeitspolitik, Industrielle Beziehungen, Soziale Ungleichheit e-mail: scheier@wzb.eu

Philipp Schulte-Braucks, Bachelor Sozialwissenschaften, Studentischer Mitarbeiter der Abteilung Ausbildung und Arbeitsmarkt am WZB.

e-mail: schulte-braucks@wzb.eu Heike Solga, Prof. Dr., Direktorin der Abteilung Ausbildung und Arbeitsmarkt am WZB und Professorin für Soziologie mit dem Schwerpunkt Arbeit, Arbeitsmarkt und Beschäftigung an der Freien Universität Berlin. Arbeitsschwerpunkte: Arbeitsmarkt, Bildung und Ausbildung. e-mail: solga@wzb.eu 
den zehn „Vorreiterbetriebe“ identifiziert, die Lösungen für das Problem fehlender zeitlicher Weiterbildungsressourcen gefunden haben. Im Rahmen von (kurzen) Fallstudien sind die unterschiedlichen betrieblichen Lösungsansätze ebenso erfasst worden wie grundlegende Merkmale ihrer Entstehungskontexte. Die Ergebnisse dieses ersten Analyseschrittes bilden den Mittelpunkt des vorliegenden Artikels. Für die folgenden Analyseschritte (die noch nicht abgeschlossen sind) wurden fünf der zehn Betriebe für ausführlichere Befragungen ausgewählt (intensive Fallstudien). Ziel dieser vertiefenden Untersuchung ist es, den entscheidenden Kontextbedingungen und Wirkungszusammenhängen auf die Spur zu kommen, die zur erfolgreichen Einführung und Nutzung der neuen Handlungsansätze beigetragen haben. In einem dritten und letzten Analyseschritt werden die zentralen Befunde aus den qualitativen Fallstudien anhand von repräsentativen Betriebsdaten (unter anderem des Instituts für Arbeitsmarkt- und Berufsforschung (IAB)) quantitativ überprüft. Darüber hinaus werden die Befunde im Rahmen eines Expertenaustauschs mit niederländischen und schwedischen Wissenschaftlern auch in einem internationalen Vergleich diskutiert.

Im Folgenden werden ausgehend von Ergebnissen der bisherigen Forschung zunächst grundlegende Barrieren der betrieblichen Weiterbildung dargestellt (Abschnitt 2). Im Anschluss daran wird das Sample von zehn „Vorreiterbetrieben“ vorgestellt, das sich - trotz dieser Barrieren - durch präventive Handlungsansätze der Arbeitszeitgestaltung und Weiterbildungspolitik auszeichnet (Abschnitt 3). Wie diese Handlungsansätze Zeit für betriebliche Weiterbildung bereitstellen, wird in Abschnitt 4 dargestellt. Die Beweggründe für deren Einführung werden nachfolgend kurz skizziert (Abschnitt 5). Der Beitrag schließt mit zwei Schlussfolgerungen zu Auswegen aus dem "Zeitdilemma“ der Weiterbildung ab (Abschnitt 6).

\section{Fehlende zeitliche Ressour- cen als Problem der be- trieblichen Weiterbildung}

Die hohe Bedeutung, die der betrieblichen Weiterbildung in der Diskussion um das lebenslange Lernen, den Erhalt der Beschäftigungsfähigkeit und die Deckung des Fachkräftebedarfs beigemessen wird, steht in einem deutlichen Kontrast zur tatsächlichen Weiterbildungsaktivität auf Seiten der Betriebe wie der Beschäftigten. Nach Daten des IAB hat 2009 weniger als die Hälfte der Betriebe Weiterbildungsmaßnahmen zeitlich oder finanziell gefördert. Nur ein Fünftel der Betriebe hat dies von 2000 bis 2008 kontinuierlich (also jedes Jahr) getan. Die Weiterbildungsbeteiligung der Beschäftigten lag 2009 nur bei etwa einem Viertel (Bechmann et al. 2010). Bestimmte Gruppen wie gering qualifizierte oder weibliche Beschäftigte mit Betreuungs- oder Pflegeverpflichtungen sind dabei besonders selten vertreten (Expertenkommission 2004; Gillen et al. 2010). Auch die Wirtschafts- und Finanzkrise 2008/09 hat sich eher einschränkend als förderlich auf die betriebliche Weiterbildung ausgewirkt (Bogedan 2010), da zeitliche Gestaltungsspielräume für Beschäftigte gefährdet wurden (Giesecke/Wotschack 2009). Die aktuelle Forschung weist auf eine Reihe grundlegender Probleme und Barrieren hin, die in der Praxis häufig zusammenwirken und die Verbreitung und Nutzung betrieblicher Weiterbildungsangebote einschränken (vgl. Abschnitt 2.1 bis 2.4).

Viele dieser Probleme lassen sich als Ausdruck eines grundlegenden Transaktionskostenproblems interpretieren, das mit der Nutzung langfristig angelegter Instrumente der Arbeitspolitik verbunden ist: Die Kosten für Weiterbildung (in Form von Zeit- und Geldinvestitionen) müssen bereits in der Gegenwart getragen werden, während der zukünftige Nutzen für Betriebe wie Beschäftigte unsicher ist. Bei Betrieben und Beschäftigten, bei denen die verfügbaren Ressourcen gering sind und Bildungsinvestitionen mit noch größeren Unsicherheiten verbunden sind, stellt Weiterbildung somit besonders häufig ein Problem dar. Dieses Grundproblem hat eine Reihe von Konsequenzen, die im Folgenden skizziert werden.

\subsection{KURZFRISTIG ORIENTIERTES BETRIEBLICHES HANDELN}

Das dominante betriebswirtschaftliche Denken ist eher von kurzfristigen Kosten-Nutzen-Kalkülen geleitet (Dobischat/ Seifert 2007, S. 106). Die Bezugsgröße für Kosten und Erträge ist damit nicht der gesamte Erwerbsverlauf der Beschäftigten, da herkömmliche Ansätze der Personalarbeit die Personalentwicklung (inklusive Weiterbildung) oft auf die erste Hälfte der Erwerbsbiografie reduzieren, an deren Ende ein sogenanntes "Career Plateau“ angenommen wird (vgl. Klammer 2008, S. 51). Benachteiligte Gruppen, wie gering qualifizierte oder weibliche Beschäftigte mit Betreuungsaufgaben, sind zudem in der Regel kaum im Fokus der Personalarbeit. Langfristige Kosten und Risiken, die durch Einschränkungen des Leistungsvermögens, Qualifizierungsdefizite und übermäßigen Verschleiß entstehen, werden in diesen Kalkülen nicht berücksichtigt. Vielmehr wird versucht, diese Kosten durch das vorzeitige Ausscheiden „angeschlagener" Mitarbeiterinnen und Mitarbeiter sowie Neurekrutierungen am Arbeitsmarkt zu externalisieren. Im Zuge der demografischen Entwicklungen wird dies jedoch für die meisten Betriebe langfristig immer weniger möglich sein. Eine zwangsläufige Überwindung dieser Kurzfristigkeit ist damit jedoch nicht verbunden, da sich die betriebliche Unsicherheit hinsichtlich des langfristigen Ertrags von Weiterbildungsinvestitionen nicht reduzieren muss, sondern durch eine erhöhte Arbeitsmarktmobilität der Beschäftigten (z.B. aufgrund attraktiver externer Jobangebote bei Fachkräftemangel) sogar erhöhen könnte.

\subsection{ZUGANGSBARRIEREN DURCH ZEITNOT}

Auf Seiten der Beschäftigten entstehen oft gerade bei den Gruppen zeitliche $\mathrm{Zu}$ gangsbarrieren, die einen besonders hohen Bedarf an Weiterbildung aufweisen (Wotschack 2010; Solga 2005). Durch die fortschreitende Intensivierung und Flexibilisierung der Arbeit einerseits sowie einen wachsenden Anteil an Zweiverdienerhaushalten und neuen Betreuungsund Pflegeaufgaben andererseits hat sich die Abstimmung beruflicher und außerberuflicher Anforderungen erheblich kompliziert (Klenner/Pfahl 2009; Keck 


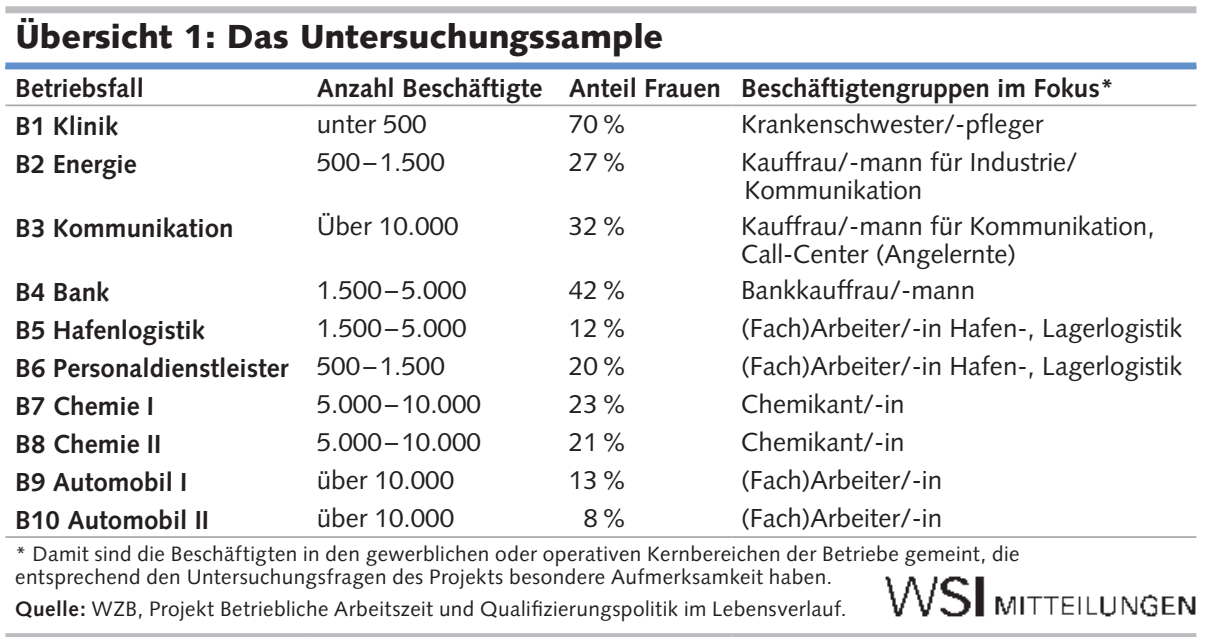

2011). Unter diesen Bedingungen werden die Sicherung eines ausreichenden Haushaltseinkommens sowie der Erhalt der täglichen Balance von beruflichen und außerberuflichen Aktivitäten für einen großen Teil der Beschäftigten gefährdet (Jurczyk 2010). Beides lässt insbesondere für weibliche Beschäftigte und niedrige Qualifikations- und Einkommensgruppen wenig zeitlichen Spielraum für Weiterbildung (Expertenkommission 2004; Gillen et al. 2010).

\subsection{KONKURRENZ UM ZEITLICHE RESSOURCEN}

Auf Seiten der Betriebe besteht ein starkes Interesse an einer möglichst flexiblen Gestaltung der Arbeitszeiten in Abhängigkeit von kurz- oder mittelfristigen Markt- und Auftragsschwankungen (Groß/Schwarz 2007; Seifert 2010b). Mehr als ein Drittel der Personalverantwortlichen gibt als Grund für fehlende betriebliche Weiterbildungsaktivitäten an, dass die Freistellung von Beschäftigten aus zeitlichen Gründen unmöglich sei (Lenske/Werner 2009; Dobischat/Seifert 2007, S. 107). In Phasen hoher Auslastung wird Zeit zur knappen Ressource, die sich nachteilig auf die betriebliche Weiterbildung auswirkt; in Flautezeiten ist die Ressource Zeit zwar (eher) verfügbar, aber die finanziellen Ressourcen des Betriebes können zu einem verknappenden Faktor werden (Seifert 2010a). Mit dem Auslaufen der staatlich geförderten Altersteilzeit und der Anhebung des gesetzlichen Renteneintrittsalters ist zudem eine starke Ausrichtung der betrieblichen Arbeitspolitik auf die Alterssicherung und den vorzeitigen Ausstieg aus dem Erwerbsleben zu beobachten (Wotsch- ack et al. 2008). Solche „nachsorgenden“ Handlungsansätze, die sich auf die letzte Erwerbsphase beziehen, laufen damit Gefahr, in Konkurrenz zu „vorsorgenden“ Ansätzen zu treten, wodurch Ressourcen für eine präventive Arbeitszeit- und Weiterbildungspolitik verloren gehen können (Barkholdt 2007).

\subsection{FEHLENDE INTEGRATION VON ARBEITSZEIT- UND WEITERBIL- DUNGSPOLITIK}

Schließlich werden in der betrieblichen Praxis Ansätze der Arbeitszeitgestaltung und Weiterbildungspolitik überwiegend isoliert voneinander gestaltet (vgl. IW 2006). Produktive Verbindungen und mögliche Synergien, etwa durch die Verbindung von Arbeitszeitinstrumenten und Qualifizierungsprogrammen, werden in der Regel verschenkt (vgl. Bahnmüller 2007; Lott/Spitznagel 2010; Seifert 2010a, S. 11). Unter den Vereinbarungen zu Arbeitszeitkonten sieht nur eine kleine Anzahl die Nutzung dieser Zeit für Bildungszwecke explizit vor (Heidemann 2008, S. 5). Auch hinsichtlich betrieblicher Langzeit- oder Lernzeitkonten wird das Potenzial für Lebenslanges Lernen in der Praxis meist blockiert (Hildebrandt et al. 2009; Seifert 2010b). Diese Integration könnte jedoch zu einer Reduzierung der oben genannten Transaktionskostenprobleme beitragen, sofern durch eine Verbindung von Qualifizierungs- und Arbeitszeitpolitik ausreichend Zeit für Weiterbildung bereitgestellt wird. Eine solche Ausrichtung der betrieblichen Arbeitspolitik bezeichnen wir als „präventiv“, da sie den gesamten Lebensverlauf von Beschäftigten in den Blick nimmt und langfristige Kosten und Risiken, die durch Qualifizierungsdefizite oder hohe Arbeitszeitbelastungen zu entstehen drohen, reduziert.

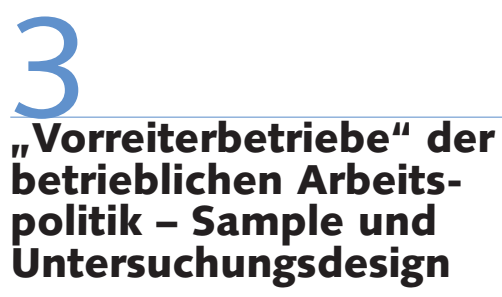

Vor dem Hintergrund der skizzierten zeitlichen Barrieren stellt sich die Frage, wie Betriebe trotz dieser Barrieren sicherstellen können, dass ausreichend Zeit für betriebliche Weiterbildung bereitgestellt wird. Wichtige Hinweise lassen sich dabei aus den Beispielen von Betrieben gewinnen, die entgegen der oben beschriebenen Tendenzen eine präventive, d.h. vorausschauende Arbeitspolitik verfolgen. Dieses Vorgehen bildet (wie in Abschnitt 1 dargestellt) den Ausgangspunkt des Projekts „Betriebliche Arbeitszeit- und Qualifizierungspolitik im Lebensverlauf“ am Wissenschaftszentrum Berlin.

Ausgehend vom methodischen Ansatz der exemplarisch vertiefenden Fallstudienforschung (vgl. Pongratz/Trinczek 2010) wurden von April 2010 bis Juni 2011 Fallstudien in Betrieben mit Vorreitercharakter in der Arbeitszeit- und Weiterbildungspolitik durchgeführt. Für die Auswahl der Betriebe war entscheidend, dass sie eine präventive Ausrichtung ihrer Arbeitszeitund Weiterbildungspolitik aufweisen, die auch gering qualifizierte und weibliche Beschäftigte im operativen und gewerblichen Bereich einbezieht und während der Krise 2008/09 beibehalten wurde. Durch umfassende Recherchen und Vorgespräche wurden dreißig potenzielle Vorreiterbetriebe ausgewählt. Aus diesen dreißig Betrieben wurden zehn Betriebe selektiert, die die oben genannten Kriterien am besten erfüllen. Dabei wurde zugleich eine Varianz hinsichtlich der Größe, Beschäftigtenstruktur und Wirtschaftssektoren sichergestellt (Übersicht 1). In allen Betrieben verfügen die Beschäftigten in der Regel über unbefristete Arbeitsverträge, auch wenn im Rahmen von Neueinstellungen für ein bis zwei Jahre zum Teil befristete Arbeitsverträge genutzt werden. Der Anteil von Zeitarbeitern und -arbeiterinnen ist gering.

In allen zehn Betrieben wurden kurze Fallstudien bestehend aus Dokumenten- 
analysen sowie ausführlichen Expertengespräche mit Vertretern und Vertreterinnen der Personalleitung und des Betriebsrates durchgeführt. In fünf der Betriebe erfolgten darüber hinaus vertiefende, intensive Fallstudien, die weitere Interviews mit betrieblichen Experten, Führungskräften und Beschäftigten umfassten.

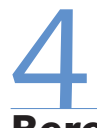

\section{Bereitstellung von Zeit für Weiterbildung - betrieb- liche Lösungsansätze}

Innerhalb dieses Samples der zehn „Vorreiterbetriebe" lassen sich systematisch charakteristische Handlungsansätze der Arbeitszeitgestaltung unterscheiden, die mehr Zeit für betriebliche Weiterbildung ermöglichen (Übersicht 2). Dabei ist hervorzuheben, dass sich die folgenden Befunde auf die Ebene der Handlungsansätze beziehen und (noch) nicht auf die tatsächliche Nutzung dieser Optionen durch die Beschäftigten. Es geht allerdings nicht um Ansätze, die nur in den Außendarstellungen der Betriebe existieren oder allein auf privilegierte Beschäftigtengruppen zielen. In allen befragten Betrieben bilden Beschäftigte in den gewerblichen oder operativen Bereichen eine wichtige Zielgruppe der arbeitspolitischen Handlungsansätze. Dieser Eindruck bestätigt sich sowohl in den betrieblichen Statistiken zur Weiterbildungsbeteiligung und Nutzung bestehender Arbeitszeitinstrumente, als auch in den bisher durchgeführten Beschäftigteninterviews. In einigen Betrieben sind Geringqualifizierte, Beschäftigte mit Migrationshintergrund oder Beschäftigte mit hohen Belastungen in- und außerhalb der Arbeit sogar explizit im Fokus der Arbeitspolitik.

Zeiträume für Weiterbildung werden in den untersuchten Betrieben in zweifacher Hinsicht im Rahmen der Arbeitspolitik gestaltet:

- direkt, indem von betrieblicher Seite explizit Zeiten für Weiterbildung definiert und bereitgestellt werden; und

- indirekt, indem durch Möglichkeiten zur besseren Anpassung der Arbeitszeit an die im Erwerbsverlauf wechselnden außerberuflichen Anforderungen auch neue zeitliche Spielräume für Weiterbildung ent-

Übersicht 2: Handlungsansätze der Arbeitszeitgestaltung

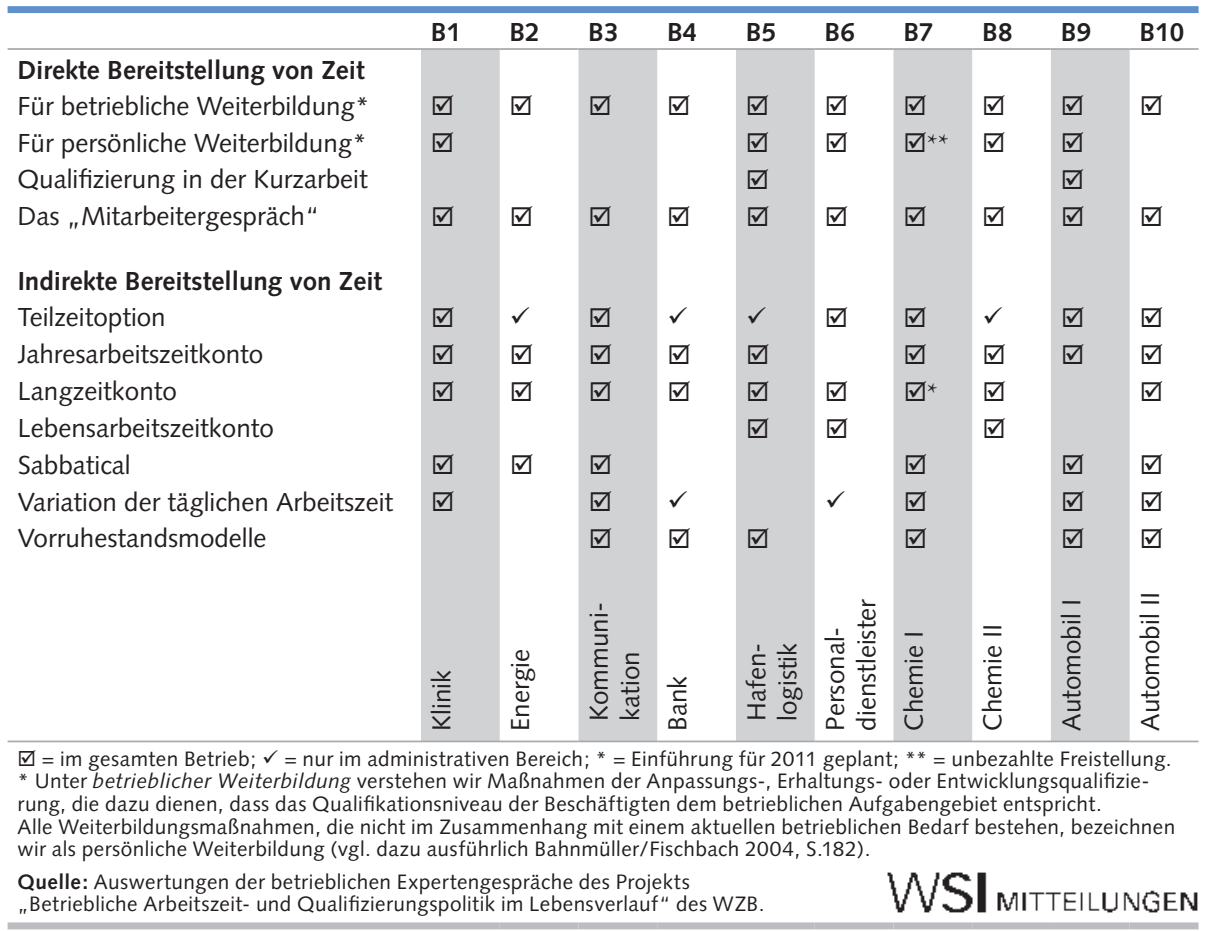

stehen (vgl. Wotschack 2010; Seifert 2010a; Scheier/Hildebrandt 2010).

Dabei lassen sich unterschiedliche Lösungen für die Überwindung der eingangs skizzierten Barrieren und Problemfelder - sowohl bei den direkten wie indirekten Lösungen - unterscheiden.

Folgende Handlungsansätze stellen direkt Zeit für betriebliche Weiterbildung zur Verfügung und tragen damit zur Integration der betrieblichen Arbeitszeitgestaltung und Weiterbildungspolitik bei (siehe Abschnitt 2.4).

(1) Bezahlte und unbezahlte Freistellungen für Weiterbildung: In allen befragten Betrieben bestehen im Zusammenhang mit Weiterbildung Regelungen zur arbeitszeitlichen Freistellung der Beschäftigten. Betrieblich notwendige Qualifizierungen werden durch bezahlte Freistellungen für die Beschäftigten ermöglicht. Dieser Befund ist nicht selbstverständlich. Untersuchungen zeigen, dass viele Betriebe selbst in diesen strategisch wichtigen Bereichen zu wenig Weiterbildung durchführen (vgl. Bahnmüller/Fischbach 2004). In den untersuchten Betrieben besteht darüber hinaus für weitergehende Weiterbildungsinteressen, wie berufliche Qualifizierung (etwa für den Meisterbrief oder ein Studium) oder persönliche Entwicklung (etwa für Fremdsprachen oder berufliche Umorientierung), in der Regel die Möglichkeit einer unbezahlten Freistellung. In einzelnen Fällen gibt es auch für diese Maßnahmen die Möglichkeit einer finanziellen Unterstützung durch den Arbeitgeber oder zumindest ideelle Formen der Unterstützung beim Wiedereinstieg oder der innerbetrieblichen Karriereplanung.

(2) Qualifizierung während der Kurzarbeit: Zwei der untersuchten Betriebe haben die freien zeitlichen Kapazitäten im Zuge des wirtschaftlichen Abschwungs 2008/09 für die Durchführung von Weiterbildungsmaßnahmen genutzt. Dabei hat die staatliche Förderung von Weiterbildung in Verbindung mit dem Kurzarbeitergeld eine wichtige Rolle gespielt. Hervorzuheben ist das Beispiel eines Betriebs aus dem Bereich der Hafenlogistik. Dort wurde die Krise für eine groß angelegte Qualifizierungsoffensive vor allem bei den Geringqualifizierten genutzt, an der etwa ein Fünftel der Beschäftigten über einen Zeitraum von 18 Monaten teilnahm. Die Mehrheit erlangte auf diese Weise einen hafenspezifischen Berufsabschluss. Das Beispiel stellt eine Lösung für das Problem der Konkurrenz um zeitliche Ressourcen dar (siehe Abschnitt 2.3), das hier durch eine antizyklische Qualifizierungspolitik gelöst wurde. Als sehr förderlich für die schnelle Umset- 
zung der Maßnahmen erwies sich dabei die bestehende enge Zusammenarbeit mit dem hafeneigenen Fortbildungszentrum (vgl. auch Heidemann 2011).

(3) Das „Mitarbeitergespräch“: In fast allen untersuchten Betrieben werden im Rahmen von regelmäßigen (meist jährlichen) Mitarbeitergesprächen (vgl. dazu Bahnmüller/Fischbach 2004) mit den direkten Vorgesetzten die Weiterbildungsbedarfe und -interessen der Beschäftigten thematisiert und deren Realisierung und zeitliche Einbettung geplant. In einer Reihe von Betrieben sind auch individuelle Arbeitszeitwünsche Gegenstand dieser Gespräche, wodurch versucht werden kann, mögliche Zeitprobleme schon im Vorfeld zu lösen. Der Institutionalisierungs- und Formalisierungsgrad der Gespräche variiert stark zwischen den Betrieben. Auch hinsichtlich der Zusammenführung der Ergebnisse dieser Gespräche auf zentraler betrieblicher Ebene gibt es große Unterschiede.

Handlungsansätze, die indirekt mehr Zeit für Weiterbildung ermöglichen, umfassen alle Arbeitszeitinstrumente, die Beschäftigten eine Variation der Dauer, Verteilung und Lage ihrer Arbeitszeit im Erwerbsverlauf erlauben. Im Unterschied zu eher kurzfristig orientierten betriebswirtschaftlichen Kalkülen (siehe Abschnitt 2.1) ist hier der langfristige Erwerbsverlauf mit seinen wechselnden zeitlichen Anforderungen eine wichtige Bezugsgröße der Personalarbeit. Es ist evident, dass die dadurch entstehenden zeitlichen Freiräume nicht notwendig zu einem größeren Weiterbildungsengagement führen müssen. Dies zeigt sich nicht zuletzt an dem Beispiel von Teilzeitbeschäftigten, die bei der Teilnahme an betrieblicher Weiterbildung zu den unterrepräsentierten Gruppen gehören (Gillen et al. 2010). Die Wahrscheinlichkeit, dass zeitliche Spielräume tatsächlich für Weiterbildung genutzt werden, steigt mit dem Vorhandensein weiterer Ressourcen der Weiterbildung (vgl. Heidemann 2010), wie sie in den meisten der im Projekt untersuchten Betriebe gegeben sind. Insgesamt lassen sich folgende Handlungsansätze der Arbeitszeitgestaltung identifizieren:

(1) Teilzeitoptionen auch im Schichtbereich: In den Betrieben im Chemie-, Metall- wie Dienstleistungsbereich finden sich Teilzeitoptionen im Schichtsystem. Das ist insofern beachtlich, da Teilzeitarbeit im
Schichtbetrieb vielerorts als unmöglich angesehen wird. Die vorgefundenen Teilzeitoptionen zielen auf männliche und weibliche Beschäftigte in den gewerblichen oder operativen Bereichen. Für die Betriebe mit hohem Männeranteil steht dabei die Reduzierung von Arbeitszeitbelastungen und arbeitsbedingten Erkrankungen im Vordergrund, während bei den Betrieben mit hohem Frauenanteil in der Regel eine bessere Vereinbarkeit von Beruf und Familie angestrebt wird. Die Unterstützung solcher Teilzeitoptionen auch in typischerweise männlichen Berufsfeldern ist auch als ein wichtiger Schritt für die Veränderung traditioneller Erwerbsmuster zu begreifen. Bei den meisten Betrieben handelt es sich um individuelle Teilzeitoptionen. In einem Betrieb wird Teilzeitarbeit (im Bereich von 30 bis 32 Stunden pro Woche) jedoch auch als kollektives Modell angestrebt. Zum Teil bestehen dabei für die Beschäftigten im Schichtsystem sehr weitreichende Möglichkeiten, die Arbeitszeit nach Bedarf und Interesse in Form ganzer Tage oder sogar auf täglicher Basis zu reduzieren. In einem Fall stockt der Arbeitgeber die Bezüge zur Rentenversicherung auf das Niveau der Vollzeitbeschäftigung auf und verhindert damit langfristige finanzielle Benachteiligungen (vgl. Abschnitt 2.2).

(2) Langfristig ausgerichtete Arbeitszeitkontensysteme: Einige Betriebe setzen bei der lebenslauforientierten Arbeitszeitgestaltung sehr stark auf differenzierte Arbeitszeitkontensysteme, die Optionen für kurz-, mittel- und langfristige Zeitbedarfe der Beschäftigten eröffnen. Diese Instrumente zielen auf eine bessere Verteilung der Arbeitszeit im Zeitraum eines oder mehrerer Jahre bis hin zum gesamten Erwerbsverlauf (vgl. Wotschack et al. 2008). Lebensarbeitszeitkonten eröffnen die Möglichkeiten des vorzeitigen Ausstiegs aus dem Erwerbsleben und werden in einigen der untersuchten Betriebe sehr stark vom Arbeitgeber unterstützt. Jahresarbeitszeitmodelle und Langzeitkonten erlauben (abhängig von den jeweiligen Anspar- und Entnahmemodalitäten) bezahlte Freistellungen während des Erwerbsverlaufs im Umfang mehrerer Wochen oder Monate. In einigen Betrieben werden diese Freiräume explizit als Instrument zur Bereitstellung von Weiterbildungszeiten begriffen. Durch getrennte Arbeitszeitkontensysteme für Beschäftigten- und Betriebsinteressen, die wir in einem Fall finden, können Konflikte und Konkurrenzen um zeitliche Ressourcen (siehe Abschnitt 2.3) vermieden werden. In einigen Fällen sind „Minusstunden“ erlaubt. Sie ermöglichen es Beschäftigten, die über kein oder nur ein geringes Guthaben verfügen, trotzdem Zeit vom Konto zu entnehmen und dieses zu einem späteren Zeitpunkt wieder auszugleichen. Dadurch können Zugangsbarrieren durch Zeitnot (siehe Abschnitt 2.2) verringert werden.

(3) Sabbaticals und variierende Schichtlagen: In einem Betriebsfall wird sehr stark auf bezahlte Freistellungsmodelle (Sabbaticals) in Kombination mit Teilzeitoptionen gesetzt. Hier geht es um eine Kombination aus Variationen der Dauer und Verteilung der Arbeitszeit. Im Mittelpunkt stehen individuelle Sabbatical-Lösungen, die in der Regel durch angepasste Entgelte, Resturlaub und Guthaben aus dem Jahresarbeitszeitkonto erzielt werden. Auf diese Weise wird auch im gewerblichen Bereich sowie gleichfalls bei niedrigem Einkommen Spielraum für Freistellungen im Erwerbsverlauf eröffnet. In einem anderen Betriebsfall werden durch angepasste Schichtlagen Zeiträume für außerberufliche Anforderungen und persönliche Weiterbildungsinteressen geschaffen. Konkret geht es um Möglichkeiten dauerhafter Früh- oder Spätschichten an Wochentagen oder ausschließliche Wochenendschichten (mit verkürzter Arbeitszeit), um Zeit für außerberufliche Anforderungen zu gewinnen; darunter fällt explizit auch die berufliche Weiterentwicklung, wie beispielsweise ein berufsbegleitender Schulabschluss oder ein Studium. Zugangsbarrieren für niedrige Einkommensgruppen (siehe Abschnitt 2.2) können dadurch reduziert werden

Die skizzierten Handlungsansätze belegen, dass Zeit für betriebliche Weiterbildung auf sehr unterschiedlichen Wegen bereitgestellt werden kann. Ein „One Best Way“ der präventiven Arbeitszeitgestaltung ist dabei nicht zu erkennen. In der Praxis werden die jeweiligen Handlungsansätze zum Teil kombiniert; es stehen eher Bündel von Maßnahmen als einzelne Instrumente im Vordergrund. Vorsorgende Instrumente zum Erhalt der Beschäftigungsfähigkeit, wie Teilzeitoptionen oder Freistellungsoptionen im Erwerbsverlauf, finden sich dabei oft in Kombination mit nachsorgenden Instrumenten wie Lebensarbeitszeitkonten 
oder Vorruhestandsoptionen (Übersicht 2). Dadurch kann eine Konkurrenz dieser beiden Handlungsfelder (siehe Abschnitt 2.3) vermieden werden.

\section{Beweggründe der präven- tiven Arbeitspolitik}

Vor dem Hintergrund dieser Ansätze stellt sich die Frage, warum diese Betriebe eine präventive Arbeitspolitik implementiert haben. Ein Bündel von Handlungsmotivationen ist rund um das Thema „demografische Entwicklung" auszumachen. Es kann dabei zwischen einem expliziten, engen Bezug auf die demografische Entwicklung und einem impliziten, über andere (politische, betriebliche oder wirtschaftliche) Faktoren vermittelten Zusammenhang unterschieden werden. Unmittelbar zu spüren sind die demografischen Veränderungen derzeit in keinem der untersuchten Vorreiterbetriebe. In zwei Betriebsfällen werden solche Veränderungen eher als ein zukünftiges Risiko in Form eines möglichen Fachkräftemangels antizipiert, auf das im Rahmen einer aktiven demografieorientierten Präventionsstrategie reagiert werden soll. In der Mehrzahl der untersuchten Vorreiterbetriebe stehen die Beweggründe eher in einem impliziten Zusammenhang mit der demografischen Entwicklung.

Hier zeigt sich, dass die eigentlichen Motive für die präventive Ausrichtung der Arbeitspolitik aus dem Spannungsverhältnis zwischen neuen gesetzlichen Vorgaben und tradierten Strukturen der betrieblichen Arbeitsorganisation resultieren. Die Anhebung des gesetzlichen Renteneintrittsalters auf 67 Lebensjahre und die fast zeitgleich auslaufende staatliche Förderung der Altersteilzeit werden in fast allen untersuchten Betrieben als Faktoren genannt, die neue betriebliche Handlungsstrategien verlangen. Probleme werden vor allem in Bereichen und Positionen mit vorwiegend manuellen Tätigkeiten gesehen, für die eine hohe bis sehr hohe physische Belastung der Beschäftigten konstatiert wird. Dort treten bei vielen Beschäftigten gegen Ende des Berufslebens zunehmend Verschleißerkrankungen wie Rückenleiden oder chronische Schlafstörungen (insbesondere bei Schichtarbeit) auf. Darüber hinaus wurden auch hohe psychische Belastungen angeführt, die die „Schichtfähigkeit" oder sogar die generelle Einsetzbarkeit der Beschäftigten zusätzlich einschränken. Da diese Einschränkungen zumeist gegen Ende des Erwerbslebens auftreten, sind Betriebe mit einem hohen Durchschnittsalter der Belegschaft besonders stark betroffen. Bereits jetzt wird hier in einer Vielzahl der Betriebe ein zentrales Handlungsfeld der Arbeitsgestaltung und Weiterbildungspolitik gesehen, das in $\mathrm{Zu}$ kunft weiter an Relevanz gewinnen wird.

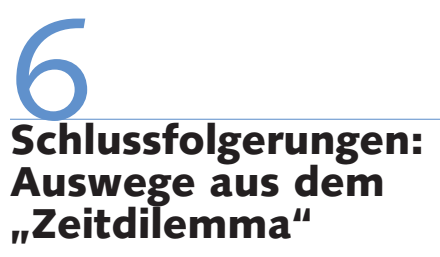

Zwei Schlussfolgerungen lassen sich aus den vorliegenden Befunden ziehen:

Erstens belegen die Ergebnisse, dass funktionierende Alternativen zur vorherrschenden betriebswirtschaftlichen Praxis bei Arbeitszeitgestaltung und Weiterbildung möglich sind. Dabei geht es nicht um „Schönwettermodelle“ oder Instrumente nur für privilegierte Beschäftigtengruppen. Die hier vorgestellten betrieblichen Handlungsansätze sind präventiv orientiert, langfristig ausgerichtet und beziehen auch typischerweise benachteiligte Beschäftigtengruppen, wie gering qualifizierte oder weibliche Beschäftigte mit Betreuungsaufgaben, ein. Das Fehlen zeitlicher Ressourcen und Spielräume stellt auf Seiten der Betriebe wie der Beschäftigten oft eine wesentliche Ursache für Weiterbildungsdefizite und Vereinbarkeitsprobleme dar. Die hier vorgestellten Beispiele belegen klar, dass es sich dabei keineswegs um unüberwindbare Barrieren oder unlösbare Probleme handelt. In den untersuchten Betrieben werden im Rahmen der Arbeitszeitgestaltung auf vielfältige Weise Zeiträume für Lebenslanges Lernen und außerberufliche Betreuungs-, Pflege- und Erholungsbedarfe geschaffen. Inwieweit diese Handlungsansätze in der Praxis tatsächlich zu einer höheren Beteiligung an Weiterbildung beitragen, kann an dieser Stelle noch nicht beantwortet werden. Auch wenn sich in den Expertengesprächen keine grundsätzlichen Zugangsbarrieren für bestimmte Beschäftigtengruppen abgezeichnet haben, finden sich, etwa in Bezug auf befristet Beschäftigte, auch
Hinweise auf Probleme und Ambivalenzen. Die tatsächlichen Nutzungsmuster der hier vorgestellten Handlungsansätze (inklusiver möglicher Selektivitäten) werden daher im weiteren Verlauf des Projekts systematisch untersucht.

Zweitens zeigen die untersuchten Betriebsfälle, dass die präventive Ausrichtung der Arbeitspolitik mit einer ganzen Reihe von betrieblichen und überbetrieblichen Voraussetzungen verbunden ist. Die untersuchten Vorreiterbetriebe weisen in ihren Problemlagen, Akteurskonstellationen $^{3}$ und institutionellen Arrangements eine große Varianz auf. Nicht einzelne Faktoren, sondern das Zusammenwirken verschiedener Bedingungen in Konstellationen prägen die betriebliche Arbeitspolitik. Die antizipierten Risiken der demografischen Entwicklung spielen dabei eine wichtige Rolle, erweisen sich aber keineswegs als hinreichende Erklärung für die präventive Ausrichtung der betrieblichen Arbeitspolitik. Auch ein akuter Fachkräftebedarf spielt in den von uns untersuchten Betrieben keine große Rolle. Wo er dennoch besteht, ist er eher von lokalen und konjunkturellen Gegebenheiten beeinflusst als von der demografischen Entwicklung. Als wichtiger Grund für eine veränderte Arbeitspolitik werden hingegen in vielen Betrieben die hohen zeitlichen, physischen und psychischen Arbeitsbelastungen mit ihren negativen Folgen für die langfristige Beschäftigungsfähigkeit genannt. Im Zusammenspiel mit den veränderten gesetzlichen Rahmenbedingungen, wie die eingeführte „Rente mit 67 Jahren" und die auslaufende Regelung zur Förderung der Altersteilzeit, entsteht hier der eigentliche Problemdruck. Dass dieser Problemdruck keineswegs automatisch und immer zu einer präventiven Ausrichtung der Arbeitspolitik beiträgt, zeigt die betriebliche Praxis. Die hier vorgestellten „Vorreiterbetriebe“ stellen heute eher Ausnahmefälle dar. Umso wichtiger ist es, die entscheidenden betrieblichen und überbetrieblichen Voraussetzungen

\footnotetext{
3 Das von der Hans-Böckler-Stiftung geförderte eigenständige Dissertationsprojekt "Schwache Interessengruppen und betriebliche Arbeitspolitik - Neue Herausforderungen und Handlungsansätze der betrieblichen Arbeitnehmervertretungen " von Franziska Scheier untersucht in diesem Zusammenhang den Einflusse verschiedener Konstellationen und Kooperationen von betrieblichen Arbeitnehmervertretungen hinsichtlich einer breiten Interessensvertretungsarbeit für die Beschäftigten.
} 
zu untersuchen, die dazu beigetragen haben, dass diese Betriebe neue Wege der Arbeitspolitik eingeschlagen haben. Diese
Aufgabe steht im Zentrum der folgenden Untersuchungsschritte des hier vorgestellten Projekts. Besondere Aufmerksamkeit hat dabei der Einfluss betrieblicher und überbetrieblicher institutioneller Arrangements und Akteurskonstellationen.

\section{LITERATUR}

Bahnmüller, R. (2007): Praxis und Perspektiven von Qualifizierungstarifverträgen. Dokumentation, 5. BIBB-Fachkongress 2007

Bahnmüller, R./Fischbach, S. (2004): Der Qualifizierungstarifvertrag für die Metall- und Elektroindustrie in Baden-Württemberg, in: WSIMitteilungen 57 (4), S. 182-189

Barkholdt, C. (2007): Altersgerechte Arbeitszeit, Gesundheitserhalt und später Ruhestand, in: Hildebrandt, E. (Hrsg.): Lebenslaufpolitik im Betrieb, Berlin, S. 119-132

Bechmann, S./Dahms, V./Fischer, A./Frei, M./Leber, U. (2010): 20 Jahre Deutsche Einheit - Ein Vergleich der west- und ostdeutschen Betriebslandschaft im Krisenjahr 2009, IAB-Forschungsbericht (6), Nürnberg

Bundesministerium für Familie, Senioren, Frauen und Jugend (BMFSFJ) (2006): 7. Familienbericht. Familie zwischen Flexibilität und Verlässlichkeit - Perspektiven für eine lebenslaufbezogene Familienpolitik, Berlin Bogedan, C. (2010): Qualifizieren statt Entlassen. Betriebliche Weiterbildung in der Krise, in: WSI-Mitteilungen 63 (6), S. 314-319

Dobischat, R./Seifert, H. (2007): Die Suche nach einer neuen zeitorganisatorischen Verteilung lebenslangen Lernens, in: Hildebrandt, E. (Hrsg.): Lebenslaufpolitik im Betrieb, Berlin, S. 105-118 Expertenkommission Finanzierung Lebenslangen Lernens (Hrsg.) (2004): Finanzierung Lebenslangen Lernens, hrsg. vom BMBF, Bielefeld Flüter-Hoffmann, C. (2010): Der Weg aus der Demografie-Falle. Lebenszyklusorientierte Personalpolitik, in: Naegele, G. (Hrsg.): Soziale Lebenslaufpolitik, Wiesbaden, S. 411-428

Giesecke, J./Wotschack, P. (2009): Flexibilisierung in Zeiten der Krise: Verlierer sind junge und gering qualifizierte Beschäftigte, WZBrief Arbeit (01), Berlin

Gillen, J./Elsholz, U./Meyer, R. (2010): Soziale Ungleichheit in der beruflichen und betrieblichen Weiterbildung, Hans-Böckler-Stiftung, Arbeitspapier (191), Düsseldorf

Groß, H./Schwarz, M. (2007): Betriebs- und Arbeitszeiten 2005, Sozialforschungsstelle Dortmund, Beiträge aus der Forschung, Bd. 153, Dortmund

Heidemann, W. (2008): Bildungszeitkonten: Betriebliche Verbreitung und Beispiele. Betriebs- und Dienstvereinbarungen. Kurzauswertungen, Archiv Betriebliche Vereinbarungen der Hans-Böckler-Stiftung, Düsseldorf

Heidemann, W. (Hrsg.) (2010): Lebenslanges Lernen im Betrieb. Neuere Praxisbeispiele, Hans-Böckler-Stiftung, Arbeitspapier 153, Düsseldorf Heidemann, W. (2011): Qualifizierung in der Kurzarbeit, Düsseldorf Hildebrandt, E./Wotschack, P./Kirschbaum, A. (2009): Zeit auf der hohen Kante. Langzeitkonten in der betrieblichen Praxis und Lebensgestaltung der Beschäftigten, Berlin

Hipp, L. (2009): Weiter mit Weiterbildung! Von der Arbeitslosen- zur Beschäftigungsversicherung, in: WSI-Mitteilungen 62 (7), S. 362-368 Institut der Deutschen Wirtschaft (IW) (2006): Projektergebnisbericht: Lebenszyklusorientierte Personalpolitik - „Work-Life-Balance“-Modelle und "Demographietools" für die betriebliche Praxis, Studie des IW im Auftrag der DekaBank, Köln
Jurczyk, K. (2010): Entgrenzte Arbeit - entgrenzte Familie, in: Groß, H./Seifert, H. (Hrsg.): Zeitkonflikte. Renaissance der Arbeitszeitpolitik, Berlin, S. 239-261

Keck, W. (2011): Pflege und Beruf - Ungleiche Chancen der Vereinbarkeit, WZBrief Arbeit (09), Berlin

Klammer, U. (2008): Business cycle and life cycle - conflicting or complementary?, in: Brandl, S./Hildebrandt, E./Wotschack, P. (Hrsg.): Arbeitszeitpolitik im Lebensverlauf, Düsseldorf

Klenner, C./Pfahl, S. (2009): Jenseits von Zeitnot und Karriereverzicht. Wege aus dem Arbeitszeitdilemma, in: Heitkötter, M./Jurczyk, K./ Lange, A./Meier-Gräwe, U. (Hrsg.): Zeit für Beziehungen? Zeit und Zeitpolitik für Familien, Opladen

Lehndorff, S. (2010): Normalität jenseits von Normen, in: Groß, H./ Seifert H. (Hrsg.): Zeitkonflikte. Renaissance der Arbeitszeitpolitik, Berlin, S. 71-99

Lenske, W./Werner, D. (2009): Umfang, Kosten und Trends der betrieblichen Weiterbildung. Ergebnisse der IW-Weiterbildungserhebung 2008, in: IW-Trends - Vierteljahresschrift zur empirischen Wirtschaftsforschung aus dem Institut der deutschen Wirtschaft Köln 36 (1), S. 51-66

Lott, M./Spitznagel, E. (2010): Präventive Arbeitsmarktpolitik: Impulse für die berufliche Weiterbildung im Betrieb, IAB-Kurzbericht (11), Nürnberg

Morschhäuser, M./Ochs, P./Huber, A. (2008): Demographiebewusstes Personalmanagement, Bertelsmann-Stiftung, Gütersloh

Pongratz, H. J./Trinczek, R. (2010): Industriesoziologische Fallstudien, Berlin

Scheier, F./Hildebrandt, E. (2010): Arbeitszeit - eine wichtig Dimension für die Lebenslaufperspektive? WZB Discussion Paper SPI 2010-506, Berlin

Seifert, H. (2010a): Arbeitszeit- und Lernzeitkonten, in: Naegele, G. (Hrsg.): Soziale Lebenslaufpolitik, Wiesbaden, S. 498-513

Seifert, H. (2010b): Beschäftigungssichernde Arbeitszeitverkürzungen, in: Groß, H./Seifert. H. (Hrsg.): Zeitkonflikte. Renaissance der Arbeitszeitpolitik, Berlin, S. 37-51

Solga, H. (2005): Ohne Abschluss in die Bildungsgesellschaft. Die Erwerbschancen gering qualifizierter Personen aus ökonomischer und soziologischer Perspektive, Opladen

Wotschack, P. (2010): Lebenslauforientierte Arbeitszeitgestaltung in Zeiten der Krise, in: Groß, H./Seifert, H. (Hrsg.): Zeitkonflikte. Renaissance der Arbeitszeitpolitik, Berlin, S. 195-212

Wotschack, P./Hildebrandt, E./Scheier, F. (2008): Langzeitkonten Neue Chancen für die Gestaltung von Arbeitszeiten und Lebensläufen?, in: WSI-Mitteilungen $61(11+12)$, S. 619-626 